\title{
Effect of Bimodal Grain Size Distribution on the Strain Hardening Behavior of a Medium-Entropy Alloy
}

\author{
Ibrahim Ondicho ${ }^{1,2}$ (C) Bernard Alunda ${ }^{3} \cdot$ Fredrick Madaraka $^{1,2} \cdot$ Melody Chepkoech $^{4}$
}

Received: 24 July 2020 / Revised: 24 September 2020 / Accepted: 28 September 2020 / Published online: 3 January 2021

(c) The Chinese Society for Metals (CSM) and Springer-Verlag GmbH Germany, part of Springer Nature 2021

\begin{abstract}
The evolution of strain hardening behavior of the $\mathrm{Fe}_{50}(\mathrm{CoCrMnNi})_{50}$ medium-entropy alloy as a function of the fraction of recrystallized microstructure and the grain size was studied using the Hollomon and Ludwigson equations. The specimens under study were partially recrystallized, fully recrystallized with ultrafine-grained microstructure, and fully recrystallized with coarse grains. The yield strength decreases steadily as the fraction of recrystallized microstructure and grain size increases due to the recovery process and the Hall-Petch effect. Interestingly, the bimodal grain distribution was found to have a significant impact on strain hardening during plastic deformation. For instance, the highest ultimate tensile strength was exhibited by a $0.97 \mu \mathrm{m}$ specimen, which was observed to contain a bimodal grain distribution. Furthermore, using the Ludwigson equation, the effect of the bimodal grain distribution was established from the behavior of $K_{2}$ and $n_{1}$ curves. These curves tend to show very high values in the specimens with a bimodal grain distribution compared to those that show a homogenous grain distribution. Additionally, the bimodal grain distribution contributes to the extensive Lüders strain observed in the $0.97 \mu \mathrm{m}$ specimen, which induces a significant deviation of the Hollomon equation at lower strains.
\end{abstract}

Keywords Metals and alloy $\cdot$ Mechanical properties $\cdot$ Microstructure $\cdot$ High-entropy alloys $\cdot$ Plastic deformation

\section{Introduction}

Conventionally, alloy design has been confined to the edges of the phase diagram while the central region has remained unexplored for a long time. This design approach is due to the existing classical physical metallurgy knowledge that elucidates the possibility of developing alloys with complex and brittle microstructures of little or no practical use

Available online at http://www.link.springer.com/journal/40195

Ibrahim Ondicho

ibrahim.ondicho@dkut.ac.ke

1 Department of Mechanical Engineering, Dedan Kimathi University of Technology, Private bag-10143, Dedan Kimathi, Nyeri, Kenya

2 Materials, Design, and Manufacturing (MADEMA) Group, Dedan Kimathi University of Technology, Private Bag-10143, Dedan Kimathi, Nyeri, Kenya

3 Department of Mining and Minerals Processing Engineering, Taita Taveta University, P.O. Box 635-80300, Voi, Kenya

4 Department of Mechanical Engineering, Howard University, 2300 6th Street NW, Washington, DC, USA
[1]. However, recently a new class of metallic materials called high-entropy alloys (HEAs) was proposed and has attracted significant research attention from material scientists and engineers [2,3]. These alloys have a different design approach such that they have more than one principal element, and their microstructures have neither distinct matrix nor solute. Interestingly, they crystallize into simple crystal structures such as face-centered cubic (FCC), bodycentered cubic (BCC), and hexagonally close-packed (HCP) $[4,5]$. Besides, the HEAs can be produced by the conventional techniques used for traditional alloys such as induction melting, arc-melting, powder metallurgy, and mechanical attrition without any specialized modification.

HEAs have been defined broadly as alloys that contain five or more principal elements having a concentration range of between 5 and 35 at.\%. Moreover, from the perspective of entropy, they have also been defined as alloys with a configurational entropy, $S_{\text {conf }}$ of $>1.5 R$ (where $R$ is the gas constant) while that of medium-entropy alloys (MEAs) is $R \leq S_{\text {conf }} \leq 1.5 R[6,7]$. The high configurational entropy in these alloys is among the factors contributing to the singlephase formation of HEAs even though some researchers have postulated that its effect is not that substantial $[4,8]$. 
Many studies have reported that HEAs have remarkable mechanical properties at room temperature, cryogenic conditions, and elevated temperature $[1,9,10]$. The excellent mechanical properties of some HEAs at a high temperature are attributed to the $\mathrm{L}_{2}\left(\mathrm{Ni}_{3} \mathrm{Al}\right)$ ordered $\gamma$-phases embedded in the FCC $\gamma$ matrix and $\mathrm{B} 2(\mathrm{NiAl})$ precipitates, which are obtained by addition of small amounts of $\mathrm{Ti}$ and $\mathrm{Al}$ elements $[11,12]$. This phenomenon is similar to that experienced in Ni-based superalloys and hence makes HEAs suitable candidates for high-temperature applications. In cryogenic conditions, the superior mechanical properties are due to observed massive mechanical twinning during plastic deformation [13].

At room temperature, various strengthening mechanisms have been studied and reported in HEAs. These mechanisms are grain boundary strengthening [14-18], solid solution strengthening, and precipitation strengthening [12, 19, 20], which are responsible for the improvement of the yield strength (YS) and on the other hand, twinning-induced plasticity (TWIP) [21-24], transformation-induced plasticity (TRIP) [24-26], and dislocation density-based strain hardening ( $\mathrm{SH}$ ), which are responsible for their impressive ultimate tensile strength (UTS). One of the empirical relations widely used to quantify SH mechanism, is the classical Hollomon equation,

$\sigma=K \varepsilon^{n}$

where $\sigma$ is the true stress, $K$ is the strengthening coefficient, $\varepsilon$ is the true strain, and $n$ is the $\mathrm{SH}$ exponent. This equation is widely used in determining the $\mathrm{SH}$ parameters of steel under different heat treatment conditions and occasionally in HEAs. However, the downside of this empirical equation is the deviation at low strains. To counter this setback, Ludwigson [27] proposed a modified version of the Hollomon equation with a term that accounts for the above deviation, where $\sigma$ is the true stress, $K_{1,} n_{1}$ and $\varepsilon$ have a similar meaning as in the Hollomon equation while $K_{2}$ and $n_{2}$ are additional constants.

$\sigma=K_{1} \varepsilon^{n_{1}}+e^{\left(K_{2}+n_{2} \varepsilon\right)}$.

On the right hand, the second term accounts for the transient flow of planar dislocations observed in low-stacking fault energy FCC metals and alloys [27]. Recently, Jeong et al. [28] successfully used the Ludwigson equation to explain the different mechanical deformation mechanisms of medium-entropy ferrous alloys. The parameters obtained from the Hollomon and Ludwigson equations are critical in predicting the formability of any alloy. For instance, the $n$ parameter plays a crucial role in the forming of sheet metal. Large $n$ values are preferred to minimize wrinkling and early fracture of metals and alloys, especially during cup drawing [29].
In our previous study of the $\mathrm{Fe}_{50}(\mathrm{CoCrMnNi})_{50}$ MEA system, a fully recrystallized specimen with ultrafine grain (UFG) size of $0.97 \mu \mathrm{m}$ showed an impressive strengthductility balance [30]. However, the reason for this impressive synergy of strength and ductility could not be established. Therefore, the present study carries out a follow-up investigation on the strain hardening behavior of the above alloy system using both the Hollomon and Ludwigson equations.

\section{Material and Methods}

Elements with purity ( $>99.8 \%$ ) were used to produce the $\mathrm{Fe}_{50}(\mathrm{CoCrFeMnNi})_{50}$ ingot (hereafter referred to as $\mathrm{Fe} 50$ ) using the arc-melting and tilt-casting technique with an initial diameter of $15 \mathrm{~mm}$. Thereafter, the ingot was homogenized at $1000^{\circ} \mathrm{C}$ for $24 \mathrm{~h}$ to remove any micro-segregation due to rapid solidification. A rectangular plate of $1.5 \mathrm{~mm}$ thick was obtained by cold rolling of the cylindrical ingot with a $90 \%$ thickness reduction. Specimens obtained from the $1.5 \mathrm{~mm}$ thick plate were annealed at $600^{\circ} \mathrm{C}$ for $5 \mathrm{~min}$, $7 \mathrm{~min}, 10 \mathrm{~min}, 30 \mathrm{~min}, 1 \mathrm{~h}, 24 \mathrm{~h}, 800^{\circ} \mathrm{C}$ for $4 \mathrm{~h}$, and $1100^{\circ} \mathrm{C}$ for $30 \mathrm{~min}$ to obtain different grain sizes. The grain size measurement was done by orientation imaging microscopy (OIM) analysis software using the scanning electron microscopy with electron backscattered diffraction (SEMEBSD) data. The low annealing temperature $\left(600^{\circ} \mathrm{C}\right)$ was chosen based on the reasons explained in our previous work [30], while the higher annealing temperatures were chosen to obtain coarse grain size. The recrystallized fraction was calculated by Image-Pro Plus analysis software using the EBSD images. The tensile test was carried out using a dogbone-shaped specimens with the above annealing conditions. The gauge length, thickness, and width of the tensile specimens were $5 \mathrm{~mm}, 1 \mathrm{~mm}$, and $2.5 \mathrm{~mm}$. The initial strain rate of $8.3 \times 10^{-4} \mathrm{~s}^{-1}$ was used. A digital image correlation technique was used for precision measurement of strain. SEM was used to observe the microstructure of annealed specimens. The specimens for microstructure characterization were prepared by mechanical polishing using the $\mathrm{SiC}$ paper and electro-polished using a 10\% perchloric acid and 90\% acetic acid solution. The Hollomon and Ludwigson equations were formulated in the Origin Pro data analysis and commercial graphing software and used for nonlinear fitting of the true stress-strain curves. 

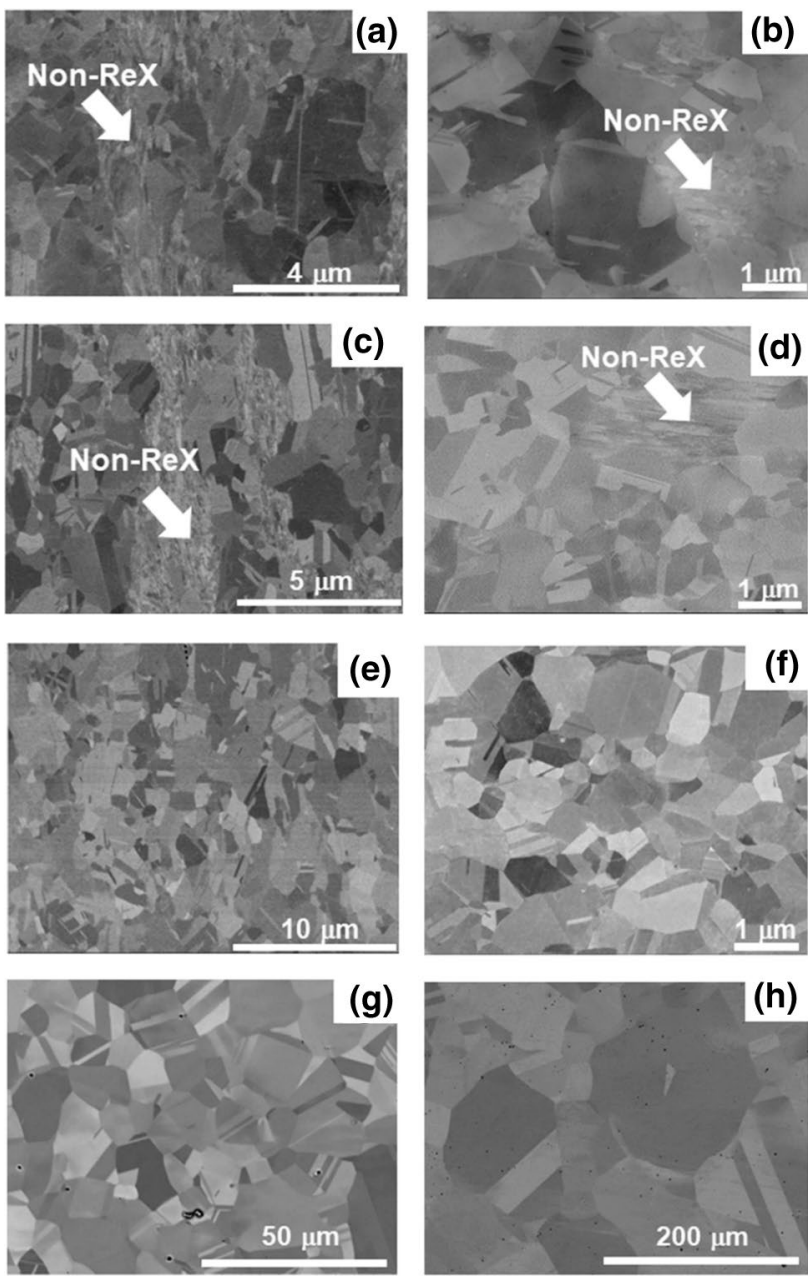

Fig. 1 Backscatter electron (BSE) images showing the microstructure of the specimens annealed at $600{ }^{\circ} \mathrm{C}$ for a $5 \mathrm{~min} \mathbf{b} 7 \mathrm{~min}$ c $10 \mathrm{~min}, \mathbf{d}$ $30 \mathrm{~min}, \mathbf{e ~} 1 \mathrm{~h}, \mathbf{f} 24 \mathrm{~h}, 800{ }^{\circ} \mathrm{C}$ for $\mathbf{g ~} 4 \mathrm{~h}$, and $1100{ }^{\circ} \mathrm{C}$ for $\mathbf{h ~} 30 \mathrm{~min}$. The white arrows in a-c represent the non-recrystallized microstructure

\section{Results}

\subsection{Microstructure}

The microstructure of the specimens annealed at different conditions is shown in Fig. 1. The specimens annealed at $600^{\circ} \mathrm{C}$ for $5 \mathrm{~min}, 7 \mathrm{~min}, 10 \mathrm{~min}$, and $30 \mathrm{~min}$ are partially recrystallized (PR) while those annealed at $600^{\circ} \mathrm{C}$ for $1 \mathrm{~h}$ and $24 \mathrm{~h}, 800^{\circ} \mathrm{C}$ for $4 \mathrm{~h}$, and $1100^{\circ} \mathrm{C}$ for $30 \mathrm{~min}$ are fully recrystallized (FR). The specimens in Figs. 1a, $\mathrm{b}, \mathrm{c}$, and $\mathrm{d}$ have a UFG size even though they are partially recrystallized. A similar phenomenon is observed in the specimens annealed at $600^{\circ} \mathrm{C}$ for $1 \mathrm{~h}$ and $24 \mathrm{~h}$ (Figs. 1e, f) where a UFG size of $0.97 \mu \mathrm{m}$ and $1.83 \mu \mathrm{m}$ is obtained. Furthermore, only a medium grain size of 7 . $40 \mu \mathrm{m}$ is achieved when a specimen is annealed at $800^{\circ} \mathrm{C}$ for $4 \mathrm{~h}$ and that it is only after exposing the Fe50 alloy to high annealing temperature of $1100^{\circ} \mathrm{C}$ for $30 \mathrm{~min}$, that a coarse-grained microstructure of $49.30 \mu \mathrm{m}$ is obtained. In addition, annealing twins can be observed in almost all the specimens regardless of the grain size, although their density per grain is higher in the recrystallized grains of the PR specimens and FR specimens with a UFG microstructure compared to those of FR specimens with coarse grains as shown in Fig. 1a, b, c, d, f, g, and h. Further microstructural analysis by EBSD as shown in Fig. 2, confirms that complete recrystallization is achieved after annealing a specimen at $600^{\circ} \mathrm{C}$ for $1 \mathrm{~h}$, however, with a bimodal grain size distribution (Fig. 2e). Klimova et al. similarly obtained a fully recrystallized microstructure of an equiatomic $\mathrm{CoCrFeMnNi} \mathrm{HEA}$ after similar annealing conditions [31].

\subsection{Mechanical Properties}

The engineering stress-strain curves of all the specimens and the Hall-Petch relationship of the fully recrystallized specimens are shown in Figs. 3a and b, respectively. Generally, it can be observed that the YS decreases as the fraction of the recrystallized microstructure increases as reported in our previous work [30] for the specimens heat-treated at $600^{\circ} \mathrm{C}$ and also as the grain size becomes coarse in specimens heat-treated at $800^{\circ} \mathrm{C}$ and $1100^{\circ} \mathrm{C}$. The latter can be attributed to the classical Hall-Petch (HP) relationship as described by Eq. 3:

$\sigma=\sigma_{\mathrm{o}}+\frac{K_{H P}}{\sqrt{d}}$.

where $\sigma$ is the stress at yield stress, $\sigma_{\mathrm{o}}$ is the friction stress, $K_{\mathrm{HP}}$ is the Hall-Petch coeffiecient, and $d$ is the mean grain size. The above relationship implies that smaller is strong and the dislocation pile-up model has been widely used to explain this phenomenon. The grain boundaries act as an impediment to dislocation motion. Therefore, dislocations tend to pileup at the grain boundary with their number and pile-up length determined by the grain size. Consequently, an adjacent grain in a coarse-grained microstructure yields easily compared to their fine-grained counterpart by initiating a dislocation nucleation due to high stress concentration at the pile-up tip [32]. The fully recrystallized specimens in the present study show a good HP relationship notwithstanding the fact that both UFG and coarse-grained specimens have been used as shown in Fig. 3b. The friction stress and HP coefficient, $K_{\mathrm{HP}}$ of $91 \mathrm{MPa}$ and $453 \mathrm{MPa}$, were obtained. It is noteworthy that the friction stress of Fe50 in the present study is less than that of CoCrNi MEA (218 MPa) reported by Yoshida et al. [33] and that equiatomic $\mathrm{CoCrFeMnNi}$ (194 MPa). This can be attributed to the less distorted crystal 

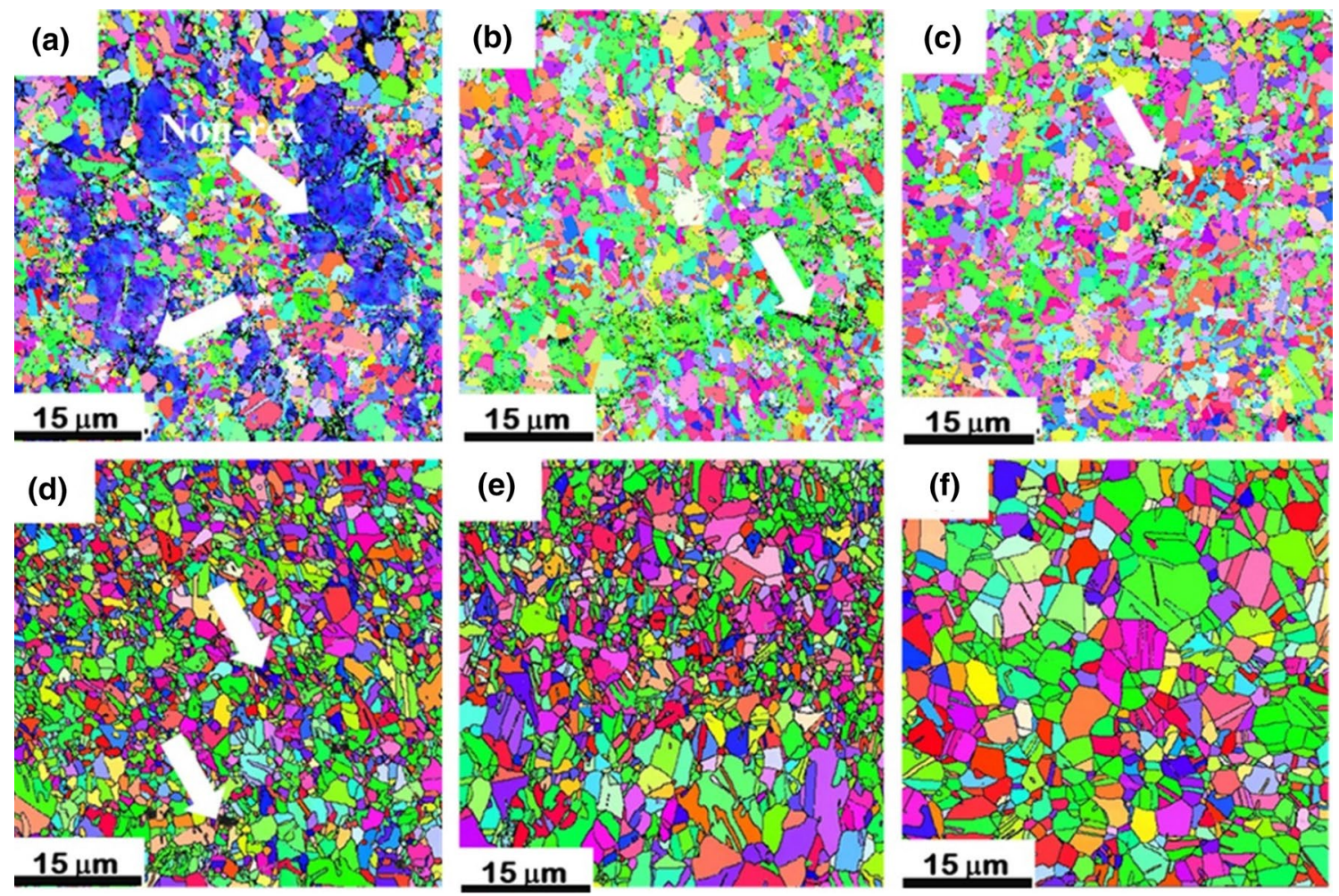

Fig. 2 EBSD images of specimens annealed for a $5 \mathrm{~min}, \mathbf{b} 7 \mathrm{~min}, \mathbf{c} 10 \mathrm{~min}, \mathbf{d} 30 \mathrm{~min}, \mathbf{e} 1 \mathrm{~h}$, and $\mathbf{f} 24 \mathrm{~h}$. The white arrows in a-d show the nonrecrystallized part of the microstructure

structure as the $\mathrm{Fe}$ content is increased from the classical equiatomic $\mathrm{CoCrFeMnNi} \mathrm{HEA} \mathrm{as} \mathrm{a} \mathrm{result} \mathrm{reduced} \mathrm{atomic}$ size misfit [34]. The HP coefficient of Fe50 is observed to be slightly less than that of equiatomic $\mathrm{CoCrFeMnNi}$ (490 MPa) and significantly more than that of the equiatomic CoCrNi (265 MPa) [33]. However, no explanation was established for this variance in the present study and as a result, a systematic study on the effect of alloy composition on the HP coefficient is currently under investigation and the results will be published in the near future.

The stress-strain curves of both PR and FR specimens are shown in Fig. 3c and their respective SH rate curves in Fig. 3d. Table 1 lists a summary of the mechanical properties of each annealing condition. The conditions mentioned above. On the one hand, it can be deduced that the YS decreases from $1020 \mathrm{MPa}$ for the PR specimen having a mean grain size of $0.42 \mu \mathrm{m}$ to $170 \mathrm{MPa}$ for the FR specimen with a mean grain size of $49.3 \mu \mathrm{m}$ according to the classical HP relationship described above. On the other hand, the uniform decrement behavior of the YS is not replicated by the UTS. Instead, the UTS decreases from $1180 \mathrm{MPa}$ for the PR specimen with a mean grain size of $0.42 \mu \mathrm{m}$ to $915 \mathrm{MPa}$ for the PR specimen with a mean grain size of $0.45 \mu \mathrm{m}$ and then increases slightly to $940 \mathrm{MPa}$ and $990 \mathrm{MPa}$ for the PR $(0.50 \mu \mathrm{m})$ and FR $(0.97 \mu \mathrm{m})$ specimens, respectively, before it decreases monotonically to $800 \mathrm{MPa}$ for the specimen with a coarse grain size of $49.3 \mu \mathrm{m}$ as shown in Table 1 .

Figure $3 \mathrm{~d}$ shows the strain hardening $(\mathrm{SH})$ rate curves of both PR and FR specimens. It can be deduced that as the recrystallized fraction and the grain size increases, the plastic instability point is postponed to higher strains according to the Considère criterion [35]. Additionally, discontinuous yielding phenomenon, evidenced by the presence of an inflection point on the $\mathrm{SH}$ rate curves, is observed in the PR specimens and FR but with UFG size specimens. The reason for the Lüders deformation, which is observed in the present study even though it is an interstitial-free alloy, is described in our previous work. [30]. Moreover, it can be observed in Fig. $3 \mathrm{~d}$ that the plastic instability point is reached easily in the PR specimens. Interestingly, the plastic instability point for the FR specimens but with UFG size (i.e., $0.97 \mu \mathrm{m}$ and $1.83 \mu \mathrm{m}$ ) is delayed to higher strains indicating their enhanced SH capability compared to their PR counterparts and that their plastic instability points are closer to those of coarse-grained specimens. This behavior can also be confirmed from their total elongation values listed in Table 1, in which their difference is not that significant from those of coarse-grained specimens, which are known to have superior SH behavior [36]. 

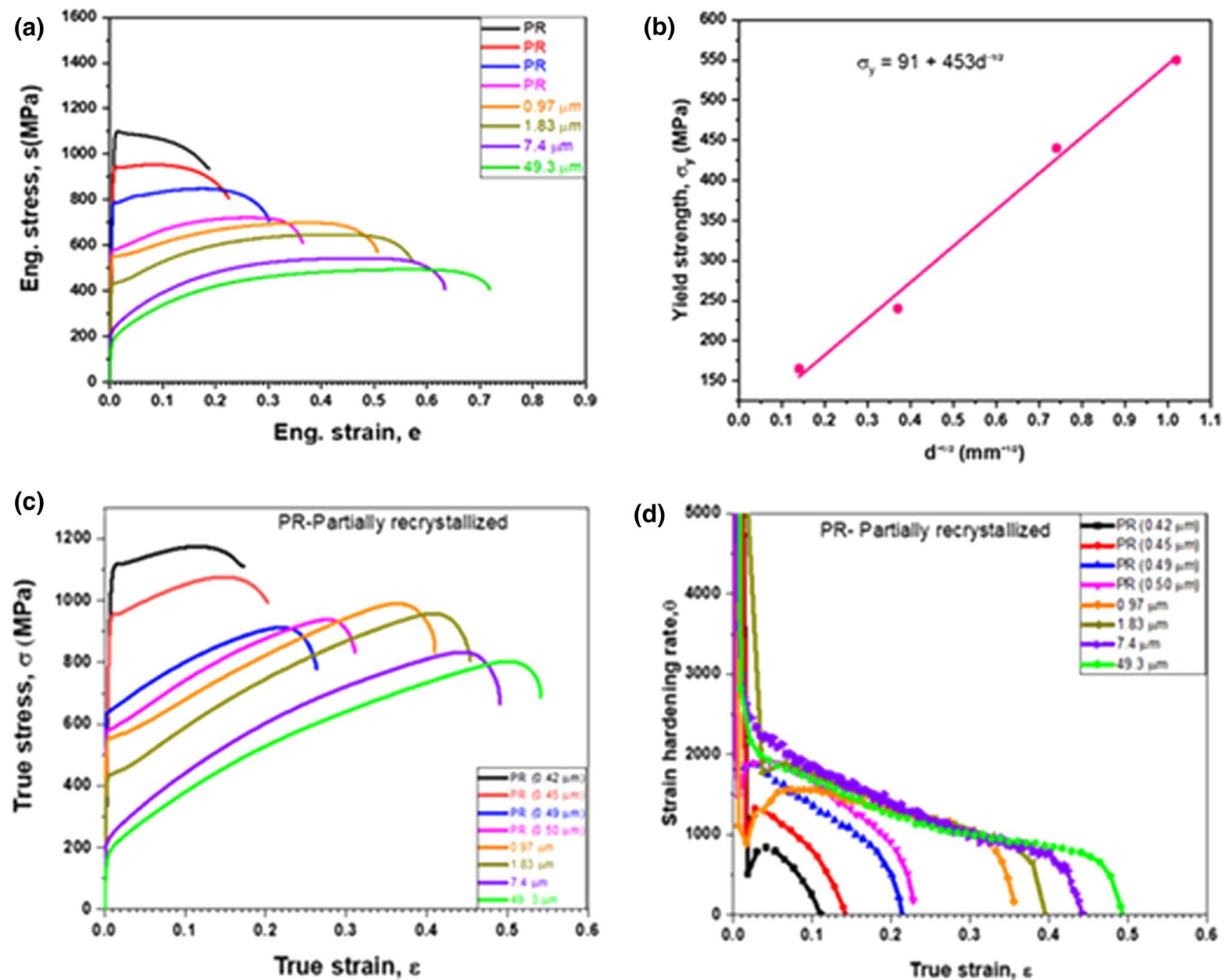

Fig. 3 a Engineering stress-strain curves of all the annealing conditions, b Hall-Petch relationship of fully recrystallized specimens, $\mathbf{c}$ true stress-strain curves $\mathbf{d}$ strain hardening rate vs. the true strain of both partially recrystallized and fully recrystallized specimens

Table 1 A summary of the grain sizes, yield strength, ultimate tensile strength, and total elongation of both partially and fully recrystallized specimens. Partially recrystallized specimens are denoted as PR, and the fully recrystallized specimens are denoted as (FR)

\begin{tabular}{|c|c|c|c|c|}
\hline Heat treatment condition & Grain size $(\mu \mathrm{m})$ & $\begin{array}{l}\text { Yield strength } \\
\text { (MPa) }\end{array}$ & $\begin{array}{l}\text { Ultimate tensile } \\
\text { strength (MPa) }\end{array}$ & $\begin{array}{l}\text { Total } \\
\text { elonga- } \\
\text { tion }(\%)\end{array}$ \\
\hline $600^{\circ} \mathrm{C} \_5 \mathrm{~min}$ & PR $(0.42$ & 1020 & 1180 & 17 \\
\hline $600^{\circ} \mathrm{C} \_7 \mathrm{~min}$ & PR (0.45) & 920 & 1070 & 20 \\
\hline $600^{\circ} \mathrm{C} \_10 \mathrm{~min}$ & $\operatorname{PR}(0.49)$ & 640 & 915 & 26 \\
\hline $600^{\circ} \mathrm{C} \_30 \mathrm{~min}$ & PR (0.50) & 580 & 940 & 31 \\
\hline $600^{\circ} \mathrm{C} \_1 \mathrm{~h}$ & 0.97 & 550 & 990 & 41 \\
\hline $600^{\circ} \mathrm{C}_{-} 24 \mathrm{~h}$ & 1.83 & 440 & 960 & 45 \\
\hline $800^{\circ} \mathrm{C}_{-} 4 \mathrm{~h}$ & 7.40 & 220 & 830 & 49 \\
\hline $1100^{\circ} \mathrm{C} \_30 \mathrm{~min}$ & 49.30 & 170 & 800 & 54 \\
\hline
\end{tabular}



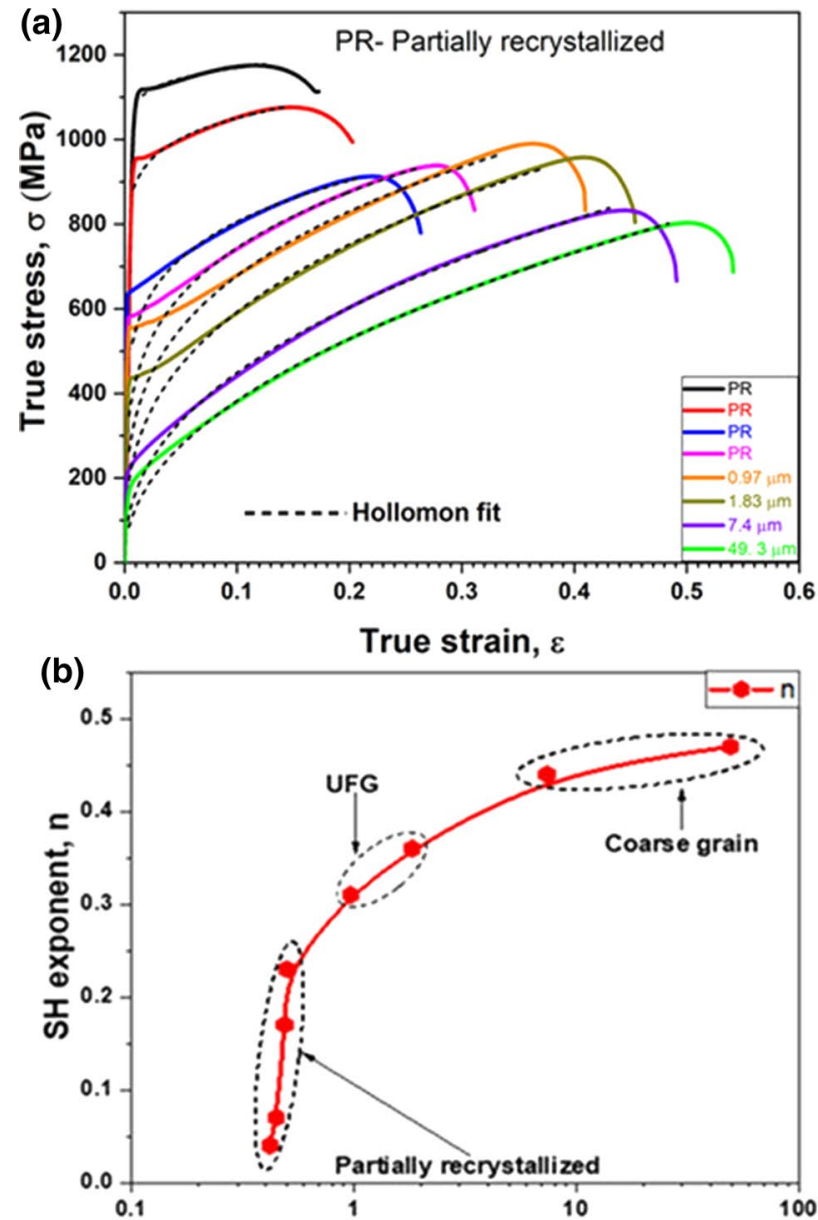

(c)

Grain size, $\mu \mathrm{m}$

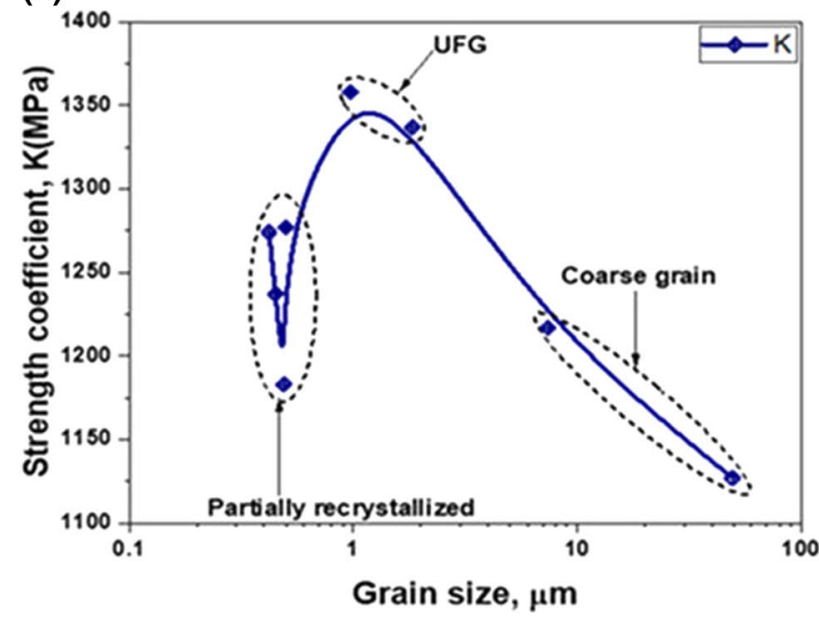

Fig. 4 a Stress-strain curve of both partially recrystallized and fully recrystallized specimens with a Hollomon fit (dotted Cyan color). b Strain hardening exponent, $n$. c Strength coefficient, $K$ versus grain size curves
Table 2 A summary of the grain sizes and strength coefficient and strain hardening exponent values from the Hollomon equation

\begin{tabular}{llll}
\hline Grain size $(\mu \mathrm{m})$ & $\begin{array}{l}\text { Recrystallized frac- } \\
\text { tion }(\%)\end{array}$ & $K$ & $n$ \\
\hline PR $(0.42)$ & 74 & 1274 & 0.04 \\
PR $(0.45)$ & 83 & 1237 & 0.07 \\
PR $(0.49)$ & 87 & 1183 & 0.17 \\
PR $(0.50)$ & 96 & 1277 & 0.23 \\
0.97 & 100 & 1358 & 0.31 \\
1.83 & 100 & 1337 & 0.36 \\
7.40 & 100 & 1217 & 0.44 \\
49.30 & 100 & 1127 & 0.47 \\
\hline
\end{tabular}

\subsection{Strain Hardening Behavior Based on the Hollomon Equation}

Figure 4a shows the stress-strain curves of both the PR and FR specimens with a Hollomon fit (dotted line with cyan color). The nonlinear curve fitting was done using a predefined Hollomon equation. It has been reported that one of the shortcomings of the Hollomon equation is the deviation that is experienced at low strains [27]. This deficiency can be observed in the present study, as shown in Fig. 4a whereby the deviation tends to increase in magnitude as the fraction of the recrystallized microstructure and Lüders strain increases, and it is most significant in the FR specimen with a UFG size of $0.97 \mu \mathrm{m}$, where it starts from a strain of approximately $10 \%$. It is important to note that this particular specimen has the most substantial Lüders strain. Consequently, the deviation tends to decrease as the grain size becomes coarse.

Figure $4 \mathrm{~b}$ and $\mathrm{c}$ shows the curves of $\mathrm{SH}$ exponent, $n$, and the strength coefficient, $K$ versus the grain size, respectively. The first curve shows three distinct regions that correspond to the level of recrystallization, as mentioned earlier. The first section corresponds to the PR specimens, where the specimen with a grain size of $0.42 \mu \mathrm{m}$ has the smallest SH exponent of 0.04, which indicates that there is limited $\mathrm{SH}$ occurring during plastic deformation. However, the SH is enhanced as the fraction of recrystallized microstructure increases, which can be inferred from the increment of the $\mathrm{SH}$ exponent to 0.23 for the PR $(0.50 \mu \mathrm{m})$ specimen. Interestingly, the SH behavior of FR specimens with UFG is greatly enhanced to 0.31 and 0.36 for the specimens with a grain size of $0.97 \mu \mathrm{m}$ and $1.83 \mu \mathrm{m}$, respectively, despite these specimens having a UFG microstructure, which has been reported to have inferior SH capacity [36]. Furthermore, the grain coarsening that occurs after the high-temperature annealing brings about a significant enhancement of SH. In particular, the 
SH exponent increases to 0.44 and 0.47 for the specimens with a grain size of $7.40 \mu \mathrm{m}$ and $49.30 \mu \mathrm{m}$, respectively, which is a noteworthy increment from the lower SH exponent values of the UFG specimens as shown in Table 2.

Furthermore, unlike the SH exponent, which increases exponentially as the fraction of the recrystallized microstructure and grain size increases, the strength coefficient,
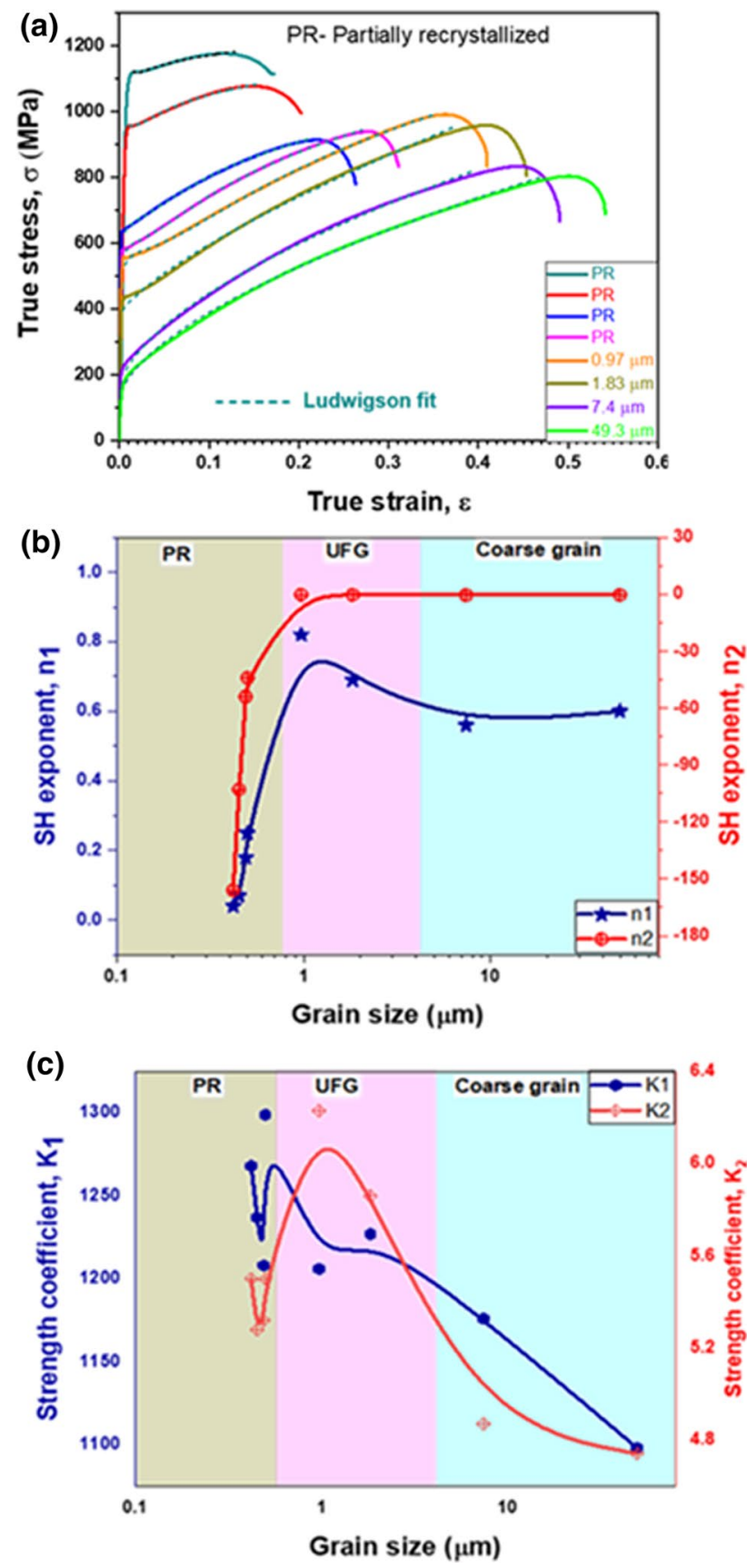

Fig. 5 a Stress-strain curve of both partially recrystallized and fully recrystallized specimens with a Ludwigson fit (dotted line with dark cyan color). b Strain hardening exponents $n_{1}$ and $n_{2}$. c Strength coefficients $\left(K_{1}, K_{2}\right)$ vs. grain size curves
$K$, shows different behavior. First, it decreases from $1274 \mathrm{MPa}$ for the PR specimen $(0.42 \mu \mathrm{m})$ to $1183 \mathrm{MPa}$ for the PR specimen $(0.49 \mu \mathrm{m})$. Secondly, it increases to $1358 \mathrm{MPa}$ for the FR recrystallized with a UFG size of $0.97 \mu \mathrm{m}$, and finally, it decreases as the grain size becomes coarse, i.e., it decreases to $1127 \mathrm{MPa}$ for the specimen with a grain size of $49.30 \mu \mathrm{m}$ as shown in Fig. $4 \mathrm{c}$, and Table 2.

\subsection{Strain Hardening Behavior Based on the Ludwigson Equation}

Figure 5a shows the stress-strain curves of both the PR and FR specimens with a nonlinear fitting using the Ludwigson equation (dotted line with red color) superimposed on them. For the fitting, the Ludwigson equation was built into the OriginPro software using the function builder since it is not predefined as it is in the case of the Hollomon equation. It is important to note that Ludwigson developed this equation to mitigate the Hollomon equation's deviation at lower strains [28]. From Fig. 5a, it is noteworthy that the Ludwigson equation achieves almost a perfect fitting with the deviations observed in the Hollomon fitting eliminated even in the specimen with a grain size of $0.97 \mu \mathrm{m}$, which exhibited the highest deviation. It is important to note that the fitting of the stress-strain curves for both the Hollomon and Ludwigson equations was done from after yielding point, which was taken as a $0.2 \%$ offset. Figure $5 \mathrm{~b}$ and c shows the curves of Ludwig parameters $\left(K_{1}, n_{1}, K_{2}, n_{2}\right)$ plotted against the grain size. The parameters $K_{1}$ and $n_{1}$ have similar significance as in the Hollomon equation whereas $K_{2}$ represents the true stress at which the plastic flow is initiated, and $n_{2}$, which is always negative, is the $\mathrm{SH}$ exponent at lower strains. The SH exponent vs. grain size curves in Fig. 5b exhibits almost a similar behavior as that of the Hollomon equation in Fig. 4b, however, with a slight variation in the $n_{1}$ curve for the FR recrystallized specimens. Generally, from the Ludwigson fitting, $n_{1}$ initially increases as the microstructure evolves from PR to FR and decreases slightly when grain

Table 3 A summary of the grain sizes and parameters from the fitting of the true stress-strain curves using the Ludwigson equation

\begin{tabular}{llllll}
\hline Grain size $(\mu \mathrm{m})$ & $K_{1}$ & $n_{1}$ & $K_{2}$ & $e^{K_{2}}$ & $n_{2}$ \\
\hline PR $(0.42)$ & 1268 & 0.04 & 5.5 & 244.69 & -156 \\
PR $(0.45)$ & 1237 & 0.07 & 5.28 & 196.37 & -103 \\
PR $(0.49)$ & 1208 & 0.18 & 5.32 & 204.38 & -54 \\
PR $(0.50)$ & 1299 & 0.25 & 5.5 & 244.69 & -44 \\
0.97 & 1206 & 0.82 & 6.23 & 507.76 & -0.09 \\
1.83 & 1227 & 0.69 & 5.86 & 350.72 & -0.22 \\
7.40 & 1176 & 0.56 & 4.87 & 130.32 & -0.36 \\
49.30 & 1098 & 0.6 & 4.74 & 114.43 & -0.3 \\
\hline
\end{tabular}


growth is initiated until a grain size of $7.40 \mu \mathrm{m}$ is achieved before it increases again when the grain size becomes coarse $(49.30 \mu \mathrm{m})$. Moreover, the FR specimen with a UFG size of $0.97 \mu \mathrm{m}$ has the highest $n_{1}$ and $n_{2}$ values at 0.82 and -0.09 (the least negative). The former represents $\mathrm{SH}$ behavior at higher strains and the latter at lower strains.

It can be deduced from Fig. $5 \mathrm{~b}$ and Table 3 that the $n_{2}$ parameter, which represents the $\mathrm{SH}$ at lower strains, follows almost a similar trend as that of $n_{1}$ by increasing as the fraction recrystallized microstructure and grain size increases. This trend is such that the specimen with the least recrystallized fraction has the most negative value, while the FR specimens with coarse grain size have the least negative values. This indicates that the $\mathrm{SH}$ at lower strains follows a similar trend as that of higher strains in the present study. Moreover, on the whole, $K_{1}$ and $K_{2}$ values tend to be high for the specimens in the PR and FR but with UFG microstructure regions, but they tend to decrease when the grain size becomes coarse.

\section{Discussion}

\subsection{Microstructure}

One of the core effects of HEAs is the sluggish diffusion effect. Tsai et al. [37] reported that the diffusion coefficients in Co-Cr-Fee-Mn-Ni alloys were lower than those of conventional alloys. Furthermore, Yoshida et al. attributed the obtained UFG microstructures in the CoCrNi MEA to the sluggish diffusion kinetics of equiatomic multicomponent alloys. In the present study, resistance to grain growth was observed in the specimens annealed at $600^{\circ} \mathrm{C}$ and $800^{\circ} \mathrm{C}$ and that substantial grain coarsening occurs only after subjecting the Fe50 alloy to annealing at $1100^{\circ} \mathrm{C}$. Therefore, this resistance to grain growth can be attributed to the low annealing temperature and probably sluggish diffusion for the case of heat treatment at $600^{\circ} \mathrm{C}$ and solely sluggish diffusion for that of $800^{\circ} \mathrm{C}[3,37]$.

\subsection{Mechanical Properties}

The high YS observed in PR specimens can be attributed to the high stress required to unlock the tangled preexisting dislocations that are introduced during the cold rolling process and the UFG size, which makes it difficult to introduce new dislocations [30, 38, 39]. Therefore, the YS decreases as more fraction of the microstructure become recrystallized, reducing the preexisting dislocations significantly, and as the recrystallized grains become coarse.

The UTS was found to exhibit a different behavior from that of the YS, in that, it decreases first as the recrystallized microstructure increases, and then it tends to increase as the specimens become FR but with UFG size before it decreases monotonically as the grain size becomes coarse. Schuh et al. [40] investigated the effect of a bimodal grain size distribution on the strength-ductility balance of nanocrystalline CoCrNi MEA. It was established that the bimodal grain microstructure enhanced both the tensile strength and the ductility of this alloy. Wang et al. [41] achieved a similar phenomenon by combining ultrafine grains with a $25 \%$ volume fraction of coarse grains in copper, in which impressive strength was obtained accompanied by excellent elongations to failure of more than $60 \%$. Two aspects were postulated to contribute to the above impressive strength-ductility balance. First, it is difficult to introduce new dislocations in the ultrafine grains of a bimodal grain microstructure, hence high yield strength, while the coarse grains are excellent in accommodating plastic deformation, leading to high ductility. Second, the confined grains exhibit a complex stress state, which significantly contributes to the $\mathrm{SH}$ of the material by inhibiting the movement of dislocations [40]. In the present study, a relatively fair distribution of bimodal grain size can be observed in the microstructure of $0.50 \mu \mathrm{m}$ and $0.97 \mu \mathrm{m}$ specimens. Nonetheless, its fraction tends to be higher in the latter specimen than in the previous specimen. Therefore, this could probably account for the sudden increment of the UTS in the specimens above before it decreases as the grain size becomes equiaxed and coarse. After $24 \mathrm{~h}$ of annealing at the same temperature, an equiaxed microstructure with a UFG size of $1.83 \mu \mathrm{m}$ is obtained. The equiaxed microstructure eases the complex stress state that is probably present in the previous specimen, hence decreasing its $\mathrm{SH}$ capability and, ultimately, the UTS [40].

\subsection{Strain Hardening Behavior Based on the Hollomon Equation}

The discontinuous yielding is a phenomenon that is widely associated with low carbon steels. It is reported to be caused by the immobilization of the dislocations at the early stages of plastic deformation due to the dislocation-carbon atom interactions, which induces dislocation pinning. However, in our previous work [30], we reported the discontinuous yielding phenomenon in interstitial-free HEAs/ MEAs, which was ascribed to the presence of the preexisting dislocations inherited from their cold rolling history. The discontinuous yielding phenomenon can also be observed in the true stress-strain curves in the present study. It is important to note that the Lüders strain increases as the fraction of the recrystallized microstructure increases, and so does the deviation of the Hollomon fit for the PR specimens. This deviation is attributed to the additional stress required for the planar slip, predominant at low strains.

Moreover, solid solution atoms, especially in concentrated alloys such as HEAs, tend to contribute to the 
resistance of glide of planar dislocations. Also, an increment of the dislocation density impedes planar dislocation motion leading to strain hardening. Therefore, the increment of the Lüders strain can be attributed to the increment of UFG microstructure in the PR specimens, which results in substantial strain hardening, which is primarily dominated by cross-slip of dislocations.

Moreover, a possible explanation for the most substantial deviation of the Hollomon fit in the FR specimen with $0.97 \mu \mathrm{m}$ is twofold. First, it is due to the presence of UFG size that has already been mentioned above, which leads to massive strain hardening in the early stages of plastic deformation. Second, this specimen contains a bimodal grain size distribution, which makes the microstructure in a complex stress state and, therefore, impeding dislocation motion. Hence, this contributes significantly to the strain hardening of this specimen, unlike in the other specimens which have a monolithic microstructure. It can be inferred from the Hollomon fit that $\mathrm{SH}$ is enhanced as the fraction of the recrystallized microstructure and the grain size increase. This is because the PR specimens with the least fraction of the recrystallized microstructure tend to have a high density of preexisting dislocations, which require minimal flow stress for their propagation and further reduces their capacity to accumulate more dislocation during plastic deformation [42-44]. Furthermore, their SH is enhanced as the fraction of the recrystallization microstructure increases, which is due to the significant reduction in the dislocation density inherited from the cold rolling process as the specimens become FR. Also, a further increment of the SH exponent, $n$ as the grain size becomes coarse, brings about enhanced $\mathrm{SH}$ of the coarse-grained specimens due to their capacity to accumulate high dislocation density during plastic straining [42].

Moreover, the $6.82 \%$ increment of the SH exponent when the grain size increases from $7.40 \mu \mathrm{m}$ to $49.30 \mu \mathrm{m}$, probably mean that there is no significant difference of the deformation and, ultimately, the strengthening mechanisms between these two specimens. The improved SH behavior observed in the coarse-grained specimens in the present study is consistent with the previous reports [36, 45], where this phenomenon is attributed to the easy activation of deformation twins due to the decreased critical twinning stress. Moreover, for the UFG microstructures, the critical twinning stress is very high; hence the twinning activity is significantly suppressed [36].

PR specimens contain preexisting dislocations, and as such, it becomes difficult to introduce new dislocations during plastic deformation. However, the density of these preexisting dislocations tends to decrease as the specimen undergoes recovery and recrystallization during the annealing process. As a result, the initial decrement of the strength coefficient, $K$ for the PR specimens, is due to the reduction of the preexisting dislocations, and the volume of their UFG microstructure is not sufficiently high enough to induce the expected strength because of their high resistance to nucleation of new dislocations. Therefore, there is an overall reduction in strength. Nonetheless, the sudden increment of strength coefficient, $K$, for the PR $(0.50 \mu \mathrm{m})$ specimen can be attributed to its predominant UFG microstructure, which was reported to be approximately $87 \%$ while the rest remained non-recrystallized. This could mean that the density of the preexisting dislocations is sufficiently low, but the large fraction of UFG size raises the resistance of this specimen to the introduction of new dislocations. This is also a possible explanation for the FR $(0.97 \mu \mathrm{m})$ specimen, which exhibits the highest value of the strength coefficient, $K(1358 \mathrm{MPa})$. Besides, the presence of the bimodal grain structure in this specimen could also be an additional source of its high value of the strength coefficient, $K$, which is likely as a result of the enhanced dislocation storage due to the complex stress state of bimodal grains and therefore, it becomes difficult to introduce new dislocations during the tensile test.

\subsection{Strain Hardening Behavior Based on the Ludwigson Equation}

The SH exponent, $n_{1}$, which represents the $\mathrm{SH}$ at higher true strains, increases as the recrystallized microstructure increases, as shown in Fig. 5b. This is similar to the behavior exhibited by the $n$ in the Hollomon fit. However, a unique phenomenon is observed in the $n_{1}$ curve of the Ludwigson fit, not observed in the $n$ curve. This phenomenon is the inflection of the $n_{1}$ curve at the FR specimen with a UFG size of $0.97 \mu \mathrm{m}$, where the value of $n_{1}$ is observed to be the highest before the curve starts to decay as the grain size coarsens and then starts to increase slightly for the coarsegrained specimen $(49.3 \mu \mathrm{m})$. This behavior shows that the FR $(0.97 \mu \mathrm{m})$ undergoes massive strain hardening more than even the most coarse-grained specimen, which is known to show impressive strain hardening behavior due to their superior dislocation storage capacity during plastic straining. Even more interesting, is the lower $n_{1}$ value of the FR specimen with a mean grain size of $1.83 \mu \mathrm{m}$, which is primarily in the same grain size regime, indicating that its strain hardening capacity is inferior to that of $0.97 \mu \mathrm{m}$ specimen. The increment of the SH exponent, $n_{1}$ values as the annealed specimens transition from PR to FR as mentioned earlier, is induced by the potential reduction of the preexisting dislocation density, which has a negative effect on their dislocation storage capacity [42, 44].

Moreover, the unprecedented high SH value exhibited by the UFG specimens $(0.97 \mu \mathrm{m}$ and $1.83 \mu \mathrm{m})$ can be attributed to the bimodal grain distribution observed in their macrostructure, which has superior dislocation storage capacity during plastic straining due to its complex stress state as 
mentioned earlier and hence leading to massive strain hardening of these specimens. Also, the SH exponent, $n_{1}$ tends to decrease as the grain size distribution becomes homogenous and coarse, which eases the stress complexity, which causes a reduction in the $\mathrm{SH}$ of the medium grain size $(7.40 \mu \mathrm{m})$ before it increases again after the grain becomes coarse $(49.3 \mu \mathrm{m})$. To further ascertain the above hypothesis, we excluded the two FR specimens that tend to show a bimodal grain distribution $(0.97 \mu \mathrm{m}$ and $1.83 \mu \mathrm{m})$ from the curves of $n_{1}$ and $n_{2}$. Interestingly, it was found that the curves exhibit a different behavior without these two specimens, as represented by the dashed lines in Fig. 5b, which is similar to that of SH exponent, $n$ in the Hollomon fit as shown in Fig. 4b. The latter curves show that the SH exponents $n_{1}$ and $n_{2}$ would increase as the microstructure transitions from a PR to FR with a coarse mean grain size, which indicates that the SH capacity of FR coarse-grained specimens is superior to their PR counterparts. This is the expected trend. This improved in the coarse-grained specimen $(49.30 \mu \mathrm{m}) \mathrm{SH}$ could be due to the activation of deformation twinning as an additional deformation mechanism due to the significantly reduced critical twinning stress as the grain size coarsens [36]. The propensity for the coarse-grained specimen $(49.30 \mu \mathrm{m})$ having deformation twin as an additional deformation mechanism was carried out in a separate study (not shown here) using the electron channeling contrast imaging (ECCI) technique. It was confirmed that after a true strain of $15 \%$, deformation twins could be observed and that no deformation twins were observed in a specimen strained to $10 \%$ of true strain. However, the observed fraction of deformation twins was not massive, and therefore, their contribution toward SH could not match that of TWIP steels and TWIP-HEAs $[46,47]$. This explains the slight increment in SH exponent $\left(n_{1}\right)$ in the coarse grain specimen, as observed in Fig. 5b, and Table 3. Furthermore, it is reasonable to attribute the sudden increment of SH in the UFG specimens to bimodal grain distribution. It is imperative to note that the bimodal grain distribution's presence does not affect the strain hardening behavior exhibited at lower strains since the shape of the $n_{2}$ curve, which represents SH at lower strains, remains the same even after removing the UFG specimens. Moreover, the $n_{2}$ curve shows a similar tendency as that of $n$ curve in the Hollomon fit, as shown in Fig. $3 b$.

Another interesting phenomenon is the similarity of the $K$ and $K_{2}$ curves in terms of their tendency, whereby they tend to be at their highest for the FR specimen with UFG microstructure $(0.97 \mu \mathrm{m})$ and after that decreases monotonically as the grain size becomes coarse. This phenomenon could be used as an additional criterion to differentiate the specimen with a bimodal grain distribution from those that have a homogeneous microstructure. However, $K_{1}$ did not show a clear tendency as that of $K$ and $K_{2}$ and that the reason for this behavior could not be established in the present study.

\section{Conclusions}

The SH behavior of the Fe50 MEA was studied as a function of the recrystallized microstructure and grain size using the Hollomon and Ludwigson equations, and the following conclusions can be inferred:

(1) As the fraction of the recrystallized microstructure and the grain size increases, the YS decreases subsequently from $1020 \mathrm{MPa}$ for the PR $(0.42 \mu \mathrm{m})$ specimen to $170 \mathrm{MPa}$ for the specimen annealed at $1100^{\circ} \mathrm{C}$ for $30 \mathrm{~min}(49.30 \mu \mathrm{m})$. This could be attributed to the recovery during recrystallization and the Hall-Petch relationship. However, the UTS initially decreases from $1180 \mathrm{MPa}$ for the PR $(0.42 \mu \mathrm{m})$ specimen to $915 \mathrm{MPa}$ for the PR $(0.49 \mu \mathrm{m})$ specimen and then it increases to $990 \mathrm{MPa}$ for the FR specimen $(0.97 \mu \mathrm{m})$ before decreasing again to $800 \mathrm{MPa}$ for the FR specimen $(49.30 \mu \mathrm{m})$. This slight increment of the UTS for the FR specimen $(0.97 \mu \mathrm{m})$ is likely due to the impressive SH caused by the presence of the bimodal grain size distribution.

(2) The characteristic deviation of the Hollomon equation at lower strains was observed after fitting the true stress-strain curves of the Fe50 MEA. The FR specimen with $0.97 \mu \mathrm{m}$ exhibited the highest deviation. This could probably be attributed to its substantial Lüders strain, which is likely caused by the bimodal grain distribution. However, in Ludwigson fitting, the above deviation was utterly absent, indicating its superiority in predicting the SH behavior of alloys using true stress-strain curves even at lower strains.

(3) According to the Ludwigson fitting, the bimodal grain distribution enhances SH in higher plastic strains and does not affect lower strains. This is supported by the SH exponent, $n_{2}$ curve, which represents $\mathrm{SH}$ at lower strains, remains the same even after excluding specimens with bimodal grain distribution $(0.97 \mu \mathrm{m}$ and $1.83 \mu \mathrm{m})$. However, the inflection point observed by the $n_{1}$ curve, which represents $\mathrm{SH}$ at higher plastic strains, disappears when the data from the two UFG specimens are excluded.

(4) The SH exponents $n_{1}$ and $n_{2}$ of the Ludwigson fitting have almost a similar tendency as that of $n$ in Hollomon fitting, where they tend to increase as the fraction of the recrystallized microstructure and as the grain size increases. The $n_{2}$ increases from the most negative value for PR specimens to the least negative values for the FR specimens. An interesting phenomenon in both cases is the high values of $n_{1}$, and $n_{2}$ observed for the FR specimen with a UFG microstructure. This phenomenon is ascribed to the probable massive $\mathrm{SH}$ 
caused by bimodal grain distribution, which is not necessarily observed in other specimens. The bimodal grain distribution is thought to enhance dislocation storage and hence enhanced $\mathrm{SH}$.

(5) Interestingly, the strength coefficients $K$ and $K_{1}$ from the Hollomon fitting and Ludwigson fitting, respectively, have a similar tendency. They tend to be moderately high for the PR specimens and low for the FR coarse-grained specimens. However, they show a significant increment for the UFG specimens with bimodal grain distribution. This phenomenon, in conjunction with the inflection point observed in the SH exponent $n_{1}$ curve, can be used as a criterion to distinguish the microstructures with and without a bimodal grain distribution.

Acknowledgements This work was supported by the National Research Foundation of Korea (NRF) grant funded by the Korea government (MSIT) (No. NRF-2019R1A2C1088535).

\section{References}

[1] M.H. Tsai, J.W. Yeh, Mater. Res. Lett. 2, 107 (2014)

[2] M.H. Tsai, Entropy 18, 252 (2016)

[3] J.W. Yeh, Ann. Chim. Sci. Des Mater. 31, 633 (2006)

[4] F. Otto, Y. Yang, H. Bei, E.P. George, Acta Mater. 61, 2628 (2013)

[5] R. Kozak, A. Sologubenko, W. Steurer, Zeitschrift Fur Krist. 230, 55 (2015)

[6] M.C. Gao, P.K. Liaw, J.W. Yeh, Y. Zhang, High-Entropy Alloys: Fundamentals App. (2016). https://doi.org/10.1007/978-3-31927013-5

[7] S. Haas, M. Mosbacher, O.N. Senkov, M. Feuerbacher, J. Freudenberger, S. Gezgin, R. Völkl, U. Glatzel, Entropy 20, 654 (2018)

[8] J.W. Yeh, JOM 65, 1759 (2013)

[9] B. Gludovatz, A. Hohenwarter, D. Catoor, E.H. Chang, E.P. George, R.O. Ritchie, Science 345, 1153 (2014)

[10] A.J. Zaddach, C. Niu, C.C. Koch, D.L. Irving, JOM 65, 1780 (2013)

[11] J.Y. He, H. Wang, H.L. Huang, X.D. Xu, M.W. Chen, Y. Wu, X.J. Liu, T.G. Nieh, K. An, Z.P. Lu, Acta Mater. 102, 187 (2016)

[12] T.T. Shun, C.H. Hung, C.F. Lee, J. Alloys Compd. 493, 105 (2010)

[13] S. Huang, H. Huang, W. Li, D. Kim, S. Lu, X. Li, E. Holmström, S.K. Kwon, L. Vitos, Nat. Commun. 9, 2381 (2018)

[14] W.H. Liu, Y. Wu, J.Y. He, T.G. Nieh, Z.P. Lu, Scr. Mater. 68, 526 (2013)

[15] R.S. Ganji, P. Sai Karthik, K. Bhanu Sankara Rao, K.V. Rajulapati, Acta. Mater. 125, 58 (2017)

[16] N. Zhou, T. Hu, J. Huang, J. Luo, Scr. Mater. 124, 160 (2016)
[17] B.R. Chen, A.C. Yeh, J.W. Yeh, Sci. Rep. 6, 22306 (2016)

[18] J. Hou, M. Zhang, S. Ma, P.K. Liaw, Y. Zhang, J. Qiao, Mater. Sci. Eng. A 707, 593 (2017)

[19] I. Toda-Caraballo, P.E.J. Rivera-Díaz-del-Castillo, Acta Mater. 85, 14 (2015)

[20] N.D. Stepanov, D.G. Shaysultanov, R.S. Chernichenko, D.M. Ikornikov, V.N. Sanin, S.V. Zherebtsov, Mater. Sci. Eng. A 728, 54 (2018)

[21] Z. Li, C.C. Tasan, H. Springer, B. Gault, D. Raabe, Sci. Rep. 7, 40704 (2017)

[22] C. Varvenne, A. Luque, W.A. Curtin, Acta Mater. 118, 164 (2016)

[23] C. Li, J.C. Li, M. Zhao, Q. Jiang, J. Alloys Compd. 475, 752 (2009)

[24] Z. Li, K.G. Pradeep, Y. Deng, D. Raabe, C.C. Tasan, Nature 534, 227 (2016)

[25] J. Li, Q. Fang, B. Liu, Y. Liu, Acta Mater. 147, 35 (2018)

[26] H. Huang, Y. Wu, J. He, H. Wang, X. Liu, K. An, W. Wu, Z. Lu, Adv. Mater. 29, 1701678 (2017)

[27] D.C. Ludwigson, Metall. Trans. 2, 2825 (1971)

[28] H.U. Jeong, N. Park, Mater. Sci. Eng. A 782, 138896 (2019)

[29] K.G. Samuel, P. Rodriguez, J. Mater. Sci. 40, 5727 (2005)

[30] M. Choi, I. Ondicho, N. Park, N. Tsuji, J. Alloys Compd. 780, 959 (2019)

[31] M.V. Klimova, D.G. Shaysultanov, S.V. Zherebtsov, N.D. Stepanov, Mater. Sci. Eng. A 748, 228 (2019)

[32] M. Kato, Mater. Trans. 55, 19 (2014)

[33] S. Yoshida, T. Bhattacharjee, Y. Bai, N. Tsuji, Scr. Mater. 134, 33 (2017)

[34] M.P. Agustianingrum, I. Ondicho, D.E. Jodi, N. Park, U. Lee, Mater. Sci. Eng. A 759, 633 (2019)

[35] R. E. Smallman, R. J. Bishop, Mod. Phys. Metall. Mater. Eng. (1999), 197-258.

[36] S.J. Sun, Y.Z. Tian, H.R. Lin, X.G. Dong, Y.H. Wang, Z.J. Zhang, Z.F. Zhang, Mater. Des. 133, 122 (2017)

[37] K.Y. Tsai, M.H. Tsai, J.W. Yeh, Acta Mater. 61, 4887 (2013)

[38] Y.Z. Tian, S. Gao, L.J. Zhao, S. Lu, R. Pippan, Z.F. Zhang, N. Tsuji, Scr. Mater. 142, 88 (2018)

[39] J. Humphreys, G. S. Rohrer, A. Rollett, in Recryst. Relat. Annealing Phenom. (2017), 375-429.

[40] B. Schuh, R. Pippan, A. Hohenwarter, Mater. Sci. Eng. A 748, 379 (2019)

[41] Y. Wang, M. Chen, F. Zhou, E. Ma, Nature 491, 912 (2002)

[42] P. Xue, B.L. Xiao, Z.Y. Ma, Mater. Sci. Eng. A 532, 106 (2012)

[43] Y. Zhao, Y. Zhu, E.J. Lavernia, Adv. Eng. Mater. 12, 769 (2010)

[44] Y.H. Zhao, J.F. Bingert, Y.T. Zhu, X.Z. Liao, R.Z. Valiev, Z. Horita, T.G. Langdon, Y.Z. Zhou, E.J. Lavernia, Appl. Phys. Lett. 92, 081903 (2008)

[45] S.J. Sun, Y.Z. Tian, H.R. Lin, H.J. Yang, X.G. Dong, Y.H. Wang, Z.F. Zhang, Mater. Sci. Eng. A 712, 603 (2018)

[46] B.C. De Cooman, Y. Estrin, S.K. Kim, Acta Mater. 142, 283 (2018)

[47] Y. Deng, C.C. Tasan, K.G. Pradeep, H. Springer, A. Kostka, D. Raabe, Acta Mater. 94, 124 (2015) 